\title{
LA NECESIDAD DE SERVICIOS DE TRADUCCIÓN E INTERPRETACIÓN EN EL SECTOR SANITARIO. LA SITUACIÓN EN LONDRES, DÜSSELDORF Y MADRID Goretti Faya Ornia \\ Universidad de Oviedo
}

\begin{abstract}
Governments constantly promote different training, work and research plans abroad. They establish links and fraternities and boost relationships with other countries in order to reach a higher international integration and cooperation. Consequently, migratory movements are more and more frequent, and they play a very important role for both development and cultural/communicative enrichment of the population of a country. However, these movements involve a continuous cultural and communicative exchange, and due to these reasons, immigrants can face many problems, the most important one of them being the communicative barrier. In this article, communicative problems that occur in health services are discussed, and a detailed study about the situation of three European cities (London, Düsseldorf and Madrid) is performed. Analyzing the immigrant populations of each country and discovering the measures (organization, requirements, procedures, workers, computing programs, among others) that each hospital uses in order to solve these problems can be very useful for translators, linguistic mediators and hospitals.
\end{abstract}

KEY WORDS: immigration, communicative problems at hospitals, linguistic mediators, translation and interpretation tools at hospitals.

\section{RESUMEN}

Los gobiernos fomentan continuamente diversos planes de formación, trabajo e investigación en el extranjero, a la vez que establecen vínculos y hermandades y potencian las relaciones con otros países para lograr una mayor integración y cooperación en el contexto internacional. Por todo ello, los movimientos migratorios son cada vez más frecuentes, y son decisivos para el desarrollo y el enriquecimiento cultural de la población de un país. No obstante, estos movimientos implican un continuo intercambio cultural y comunicativo y, por este motivo, los inmigrantes pueden tener que enfrentarse a muchas dificultades, en donde la piedra angular es la barrera comunicativa. En este artículo se tratan concretamente los problemas comunicativos que tienen lugar en los servicios de salud, y se lleva a cabo un estudio detallado sobre la situación de tres ciudades europeas (Londres, Düsseldorf y Madrid). Analizar la población inmigrante de cada país así como descubrir las medidas (organización, requisitos, procedimientos, trabajadores, programas informáticos, entre 
La necesidad de servicios de Traducción e Interpretación en el sector sanitario

otros) que emplea cada hospital para solucionar estos problemas puede resultar muy útil tanto para traductores y mediadores lingüísticos como para los propios hospitales.

PALABRAS CLAVE: inmigración, problemas comunicativos en hospitales, mediadores lingüísticos, herramientas de traducción e interpretación en hospitales.

FECHA DE RECEPCIÓN: 11/08/2014

FECHA DE ACEPTACIÓN: 25/03/2015

PÁGINAS: 543-574 


\section{INTRODUCCIÓN}

Este estudio parte de la convicción de los grandes problemas comunicativos que tienen lugar en los servicios de salud, así como de la existencia de diferentes recursos y estrategias que pone en práctica cada hospital y cada país. Los datos y conclusiones se basan en un estudio totalmente práctico, que he llevado a cabo previamente de manera bastante exhaustiva, principalmente a través de cuestionarios y por contacto directo con diversos participantes.

A pesar de que en los últimos años se ha experimentado un gran avance en los servicios de interpretación y traducción en el sector sanitario, y que se han desarrollado varias herramientas que facilitan aquellas situaciones en las que la comunicación se ve limitada, sigue sin darse una solución eficaz y global para todos los pacientes, médicos, situaciones y centros.

\section{CRITERIOS PARA EL ESTUDIO}

El presente trabajo pretende reflejar algunas de las carencias de los servicios de traducción e interpretación en los hospitales de diferentes ciudades europeas; para ello, se toman de muestra Londres (Reino Unido), Düsseldorf (Alemania) y Madrid (España).

Para conseguir dicho objetivo, este estudio eminentemente práctico parte con un análisis de la población inmigrante de cada uno de los tres países, lo que permitirá determinar si las cifras de inmigración influyen directamente en la prestación de servicios de traducción en los hospitales de la zona. Posteriormente, procederé a analizar diversos cuestionarios respondidos por pacientes, médicos, administradores de hospitales e intérpretes, que permitirán obtener una visión real y objetiva de la situación actual, es decir, los problemas reales y las soluciones que se implementan, así como los aspectos que aún requieren atención y las propuestas existentes o bien las propias experiencias de los participantes.

\section{POBLACIÓN INMIGRANTE}

Según datos obtenidos a través de estadísticas y gráficos elaborados en los últimos años por diversos organismos oficiales (UK National Statistics, Statistisches Bundesamt Deutschland y el Instituto Nacional de Estadística), puede afirmarse que a grandes rasgos la población inmigrante de estos países se estructura como se indica: 


\subsection{EL REINO UNIDO}

Según datos del Instituto Nacional de Estadística (UK National Statistics), la BBC y las embajadas, el Reino Unido tiene una población aproximada de 60975400 habitantes. De ellos, 3941000 son inmigrantes. En el área de Londres, el número de habitantes asciende a 7556 900, y el número de inmigrantes a 1558000 . Los inmigrantes provienen por lo general de Polonia, la República de Irlanda, India, los Estados Unidos y Pakistán. La población alemana ocupa el puesto décimo y la española el decimoséptimo en lo que a cifras de inmigración se refiere.

\subsection{Alemania}

Según datos del Instituto Nacional de Estadística (Detastis), las embajadas y el Ministerio del Interior (Bundesministerium des Innern), Alemania tiene una población aproximada de 82.217.837 habitantes. De ellos, 6.744.879 son inmigrantes. En la región de Renania del Norte-Westfalia, el número de habitantes asciende a 17968 124, y el número de inmigrantes a 1814 747. Los inmigrantes provienen por lo general de Turquía, Italia, Polonia, Grecia y Serbia y Montenegro. La población española ocupa el puesto decimocuarto y la británica el decimosexto.

\subsection{ESPAÑA}

Según datos del Instituto Nacional de Estadística (INE), las embajadas y el Ministerio del Interior, España tiene una población aproximada de 46157822 habitantes. De ellos, 4526522 son inmigrantes. En la Comunidad de Madrid, el número de habitantes asciende a 6271 638, y el número de inmigrantes a 1005 381. Los inmigrantes provienen por lo general de Marruecos, Ecuador, Colombia, Rumanía y Bulgaria. Destacan asimismo los inmigrantes argentinos y los procedentes de países asiáticos y de Oceanía. La población británica ocupa el puesto sexto y la población alemana el noveno.

\section{DESARROLLO DE ANÁLISIS}

Para determinar los problemas comunicativos a los que se enfrentan los inmigrantes de estas tres ciudades en el ámbito sanitario, he diseñado unos 
cuestionarios (dirigidos a pacientes, médicos, administradores de hospitales e intérpretes) y cuyas preguntas giran en torno a la propia experiencia vivida (ver sección Anexos).

A continuación, procedo a resumir las conclusiones generales extraídas del conjunto de respuestas de cada uno de los grupos, lo que nos permitirá conocer cuáles son las dificultades, cuáles son las actuales soluciones aplicadas y cuáles están aún por cubrir o mejorar.

\subsection{PACIENTES}

El cuestionario para pacientes (Anexo 1) se centra en la figura del paciente extranjero que acude al médico y desconoce o tiene vagos conocimientos del idioma del país de destino. En base a las múltiples respuestas obtenidas con este cuestionario, debo mencionar que pueden producirse varios escenarios comunicativos: a) el paciente y el médico se entienden mediante gestos y el poco conocimiento del idioma del país por parte del paciente; b) el paciente y el médico utilizan un tercer idioma que les permitirá entenderse con mayor fluidez; c) el paciente o el médico cambia al idioma de su interlocutor; d) un intérprete o mediador comunicativo (ya sea un profesional o bien un amigo, familiar o conocido del paciente) tiene que mediar en la conversación para que la comunicación sea efectiva.

Es lógico pensar que los interlocutores que presentan mayores dificultades de comunicación son los que pertenecen al primer grupo, ya que en este caso la comunicación (y más aún la comunicación especializada) se ve totalmente obstaculizada. Los idiomas participantes en estas conversaciones suelen ser muy distantes entre sí (por ejemplo, chino, búlgaro, árabe, etc.), y que suelen resultar totalmente desconocidos para uno de los dos hablantes. Asimismo, también cabe pensar que los interlocutores que presentan mayor comodidad comunicativa son los pertenecientes al cuarto grupo, ya que interviene un mediador cultural que conoce las dos lenguas y las dos culturas.

Entre las experiencias personales obtenidas mediante el cuestionario, cabe destacar que la inmensa mayoría de los informantes destaca la dificultad de comunicarse con el médico (explicar con claridad las dolencias y comprender el diagnóstico y el tratamiento) de no haber sido por la intervención de intérpretes o el dominio de un tercer idioma. Asimismo, muchos de los pacientes que no contaron con la ayuda de un mediador lingüístico afirman haber tenido que recurrir a la comunicación por gestos para poder comunicarse mejor. En su gran mayoría, se considera al intérprete una ayuda, y no se piensa que pueda suponer una violación de la intimidad. 
Entre las sugerencias que realizan, destaca la importancia de que el intérprete disponga no solo de una formación lingüística, sino también de una formación médica que le permita identificar los principales términos y conceptos médicos, ya que de este modo, podrá entender y comunicar sin dificultad. Muchos de ellos mencionan también la utilidad de materiales gráficos y diccionarios bilingües en las consultas, ya que consideran que podrían facilitar la comunicación en gran medida.

\subsection{MÉdiCOS}

Los médicos participantes (Anexo 2) en esta encuesta señalan que aunque suelen trabajar siempre con las mismas nacionalidades (las que sean más frecuentes entre los inmigrantes de la zona, según afirman), la tasa de atención a pacientes extranjeros puede variar ya que depende de circunstancias externas.

Cabe destacar que muchos de los médicos de la Unidad de Medicina Tropical del Hospital Ramón y Cajal (Madrid), que trabajan activamente con pacientes extranjeros, indican que normalmente el idioma no supone un obstáculo para ellos, ya que si no se pueden comunicar en inglés, utilizan los servicios de traducción e interpretación que ofrece el hospital. Estos servicios son frecuentes para idiomas poco conocidos y en situaciones de urgencias, cirugías y consultas.

Todos los participantes han afirmado que han encontrado muy útil este servicio y que no han percibido en ningún momento una falta de comprensión grave por parte del intérprete. Por este motivo, destacan positivamente la importancia de estos servicios para eliminar la barrera lingüística y extralingüística (diferencia entre culturas y costumbres) que se dan en numerosas situaciones.

Por último, cabe mencionar que todos ellos apuntan a una necesidad de formación del intérprete, desde un punto de vista tanto médico (medicina general) como lingüístico, para poder ofrecer servicios de traducción e interpretación de calidad. Además, como medida de mejora, proponen que se disponga de un número mínimo permanente de estos mediadores en las consultas, y que se confíe más en el trabajo que realizan, o bien que se instruya al personal sobre cómo trabajar con mediadores en las consultas, ya que algunos "colegas de profesión" muestran a veces desconfianza en el desarrollo de la conversación (quizás debido a la falta de especialización técnica de algunos intérpretes). 


\subsection{HOSPitALES}

En primer lugar, hay que destacar que según un gráfico proporcionado por el Ministerio de Sanidad sobre el gasto en servicios sanitarios de los países europeos, Alemania es el segundo país europeo que más dinero invierte (detrás de Francia), mientras que el Reino Unido y España ocupan un empatado decimosegundo puesto.

A continuación, resumo las conclusiones extraídas de los cuestionarios cumplimentados por los hospitales (Anexo 3); esto nos permitirá conocer la organización y prestación de los servicios de traducción e interpretación en cada país.

\subsubsection{El Reino Unido}

El servicio Department of Health de Londres (departamento independiente en el que no contratan directamente personal, pero que ofrece publicaciones, servicios de control y políticas sociales y sanitarias para el sistema nacional de salud, NHS) destaca que el Reino Unido es un país muy diverso desde el punto de vista étnico y que, por eso, se busca en todo momento la igualdad en el trato. Por ello, afirma que todo aquel inmigrante con dificultades lingüísticas puede optar a servicios de traducción e interpretación ya que el acceso a los servicios del NHS se basa en las necesidades clínicas del paciente y no en una cuestión de etnia.

En relación con ello, el sistema de salud británico ofrece estos servicios de traducción e interpretación en todos los casos, ya que la facilitación de la comunicación no es un aspecto extraordinario del hospital sino que así lo estipula la ley (Disability Discrimination Act 2005, Race Relations Amendment Act 2000, y la Ley de Derechos Humanos de 1998). Por tanto, si un paciente precisa de estos servicios, será responsabilidad de la sección local del servicio de salud (NHS) ofrecérselos; esto no solo afecta a la interpretación en la consulta, sino que puede extenderse incluso al ámbito de la traducción, ya sea para la traducción de folletos, prospectos, indicaciones o de cualquier otro tipo de documentos.

Sin embargo, este departamento explica que no todos los hospitales tienen que disponer de una sección de traducción e interpretación ni tienen por qué contactar directamente con una empresa privada, sino que en algunas áreas es frecuente encontrar una unión del servicio de salud y el ayuntamiento para financiar dichas actividades. Es el caso de la agencia CINTRA (Cambridgeshire Interpretation and Translation Agency) que fundó en 1995 el Gobierno y el 
Servicio de Salud, y que ofrece intérpretes y traductores a 50 idiomas para el sector público. Se trata de un organismo privado que se dedica a ofrecer servicios de traducción e interpretación, pero no es el único que existe. No obstante, si un hospital cuenta con una gran demanda y decide disponer de un departamento propio y ofrecer este servicio directamente, los costes derivados correrían a cargo de la autoridad local y no del gobierno central.

En cuanto a las cualidades que debe poseer un mediador de esta índole, Department of Health afirma que cuando el NHS necesita un intérprete, no busca solo un alto nivel de la lengua de destino, sino también la fluidez de un hablante nativo. Aunque hay varios organismos de formación para intérpretes, Department of Health propone el Institute of Linguists de Londres, que otorga el título de licenciatura o posgrado.

A continuación, expongo ejemplos concretos de la forma de trabajo y organización de algunos de los hospitales encuestados del Reino Unido:

El Guy's and St. Thomas' Hospital presta estos servicios que pueden solicitar tanto pacientes como médicos, y dispone de un presupuesto anual fijo para estos fines, de manera que el paciente no tenga que abonar nada en ningún momento. Esta cantidad se presupuesta en función de los gastos del año anterior, de las fluctuaciones de inmigrantes que llegan al Reino Unido, y se tienen en cuenta algunos proyectos que pueden requerir más dinero. Este hospital dispone de personal lingüístico en plantilla para realizar interpretaciones, pero también establecen contratos con empresas de traducción privadas o con traductores/intérpretes freelance. Se interesan no solo por la comunicación oral, sino también por la comunicación con sordos (lenguaje de signos) y ciegos (braille). No obstante, indican que la traducción bilateral (también llamada de enlace) es la más solicitada y, por tanto, a la que más dinero se destina.

El King's College Hospital invierte aproximadamente 500000 libras anules en la oferta de servicios de traducción e interpretación a 150 idiomas debido a la gran diversidad étnica de la zona. Además de traducciones e interpretaciones de enlace presenciales (que deben reservarse previamente), ofrecen servicios de interpretación telefónica las 24 horas. Informa asimismo que cada hospital del NHS proporciona sus propios servicios de interpretación y que cada uno dispone de un perfil diferente y de distintos costes asociados.

La fundación hospitalaria de Chelsea and Westminster NHS Foundation Trust (organización que presta servicios de apoyo y promoción de la salud, información y consejo) informa de que muchos hospitales suelen trabajar con la empresa Language Line (la misma que utiliza el NHS, e incluso la propia fundación). Apunta que algunos hospitales también contratan a traductores o a trabajadores que se encargan de ayudar a minorías étnicas que tienen dificultades con el inglés. Esta fundación no destina un presupuesto fijo 
a traducción e interpretación, sino que afronta estos gastos cuando son necesarios. Por último, destaca que el aspecto comunicativo es muy importante $\mathrm{y}$, en los últimos 5 años han percibido un aumento considerable en el interés por integrar a la población inmigrante.

En resumen, todos los hospitales británicos están obligados a ofrecer estos servicios de traducción e interpretación en caso de que se requiera, ya que así lo estipula la legislación vigente que trata de mantener la igualdad de condiciones de los inmigrantes. Ahora bien, en función de la demanda que tenga cada hospital (influenciada por la población inmigrante de la zona), la prestación de estos servicios se organizará de uno u otro modo. Lo más frecuente es trabajar en colaboración con la empresa privada Language Line, que es la empresa que ofrece sus servicios al NHS. No obstante, determinados hospitales con alta demanda de servicios de traducción e interpretación pueden disponer de su propio departamento de Traducción (como el caso de Guy's Hospital) o pueden contratar directamente a otra empresa privada o a traductores/intérpretes freelance. También es posible que el servicio de salud y el ayuntamiento se unan para financiar estos servicios a través de una empresa de carácter privado (el caso de CINTRA).

\subsubsection{Alemania}

La BDÜ (Bundesverband der Dolmetscher und Übersetzer, Asociación federal de Traductores e Intérpretes) me informa mediante correspondencia privada que muchos extranjeros que se instalan en Alemania provienen de Turquía, los Balcanes, Polonia, países de la antigua Unión Soviética y de países árabes, y que por tanto no dominan el idioma alemán y esto da lugar a problemas comunicativos en hospitales, pero también con las autoridades. Puesto que muchos de estos pacientes extranjeros no disponen de dinero para pagar los servicios de un intérprete, suelen acudir al médico con familiares que conozcan las dos lenguas. En otros casos, sin embargo, es necesario que les ayuden a comunicarse empleados del hospital que provengan del mismo país o conozcan su lengua. No obstante, aunque la BDÜ no puede asegurar que en Düsseldorf exista este procedimiento, sí dan fe de que en muchos hospitales es frecuente elaborar una lista en la que se especifique el nombre de los trabajadores del hospital expertos en otros idiomas, para poder recurrir a ellos en caso de que no se pueda establecer una comunicación entre paciente y médico. La BDÜ me indica asimismo que en las ciudades más grandes, como Berlín, sí que ofrecen este tipo de servicios de manera más organizada. Emplean por ejemplo la denominada "Community interpreting", término que hace referencia a la interpretación que media entre aquellos grupos de personas 
que pertenecen a comunidades de distinta lengua y cultura y que atraviesan un proceso de integración en la comunidad del país de acogida, y en consecuencia, tienen acceso a los servicios públicos de dicho país.

Por otro lado la BDÜ también me informa personalmente de que la intervención de traductores e intérpretes profesionales no está muy demandada ya que el personal en estos hospitales y clínicas es multicultural y cada vez hay más médicos que pueden comunicarse con facilidad con los pacientes gracias a algún colega de profesión. En el caso de los pacientes turcos (muy numerosos en Alemania) es especialmente frecuente que acudan al médico con familiares o amigos que les pueden ayudar. El problema surge con idiomas que resultan desconocidos para todos los médicos, en este caso, el hospital se pone en contacto con algún intérprete que pueda prestar este servicio lingüístico o de lengua de signos. Estas actividades las abonará la Krankenkasse (Caja de enfermedad).

Por último, la BDÜ también me comunica sus reflexiones sobre el tema de la traducción (ámbito escrito) y me informa de que en el ámbito nacional, la traducción propiamente dicha es muy importante y está en pleno auge, ya que los hospitales actualmente tienen la necesidad de traducir toda su documentación (normalmente en oficinas de traducción especializadas en varios idiomas) o de adquirir documentos traducidos.

De forma más concreta, la Universität Hamburg, que trabaja activamente en este tema, me informa mediante correspondencia privada que en los hospitales en los que han llevado a cabo sus estudios lingüísticos se traduce e interpreta principalmente a turco, ruso, serbio (croata y bosnio), dari, farsi, árabe y polaco, aunque puede haber diferencias importantes entre regiones y ciudades. Esta universidad afirma asimismo que, por lo general, no se necesitan interpretaciones a inglés, ya que los profesionales de la salud lo hablan sin dificultad, ni tampoco son necesarias interpretaciones a español, italiano y portugués ya que normalmente los inmigrantes con estas nacionalidades aprendieron alemán hace tiempo, puesto que emigraron hace años y viven desde entonces en Alemania. Por último, indica que tan solo en ocasiones es necesario interpretar a español y portugués para pacientes de Latinoamérica, puesto que son los inmigrantes más recientes.

Por otro lado, cabe mencionar que tras los cuestionarios enviados a cada hospital y las investigaciones pertinentes realizadas, se puede determinar que a fin de facilitar la comunicación y ahorrar costes en las interpretaciones que se llevan a cabo en los servicios de salud, se han desarrollado diferentes programas e instrumentos. Se trata, por ejemplo, del software para PDA (arpadi $($ ) que sirve para comunicar a los pacientes con los médicos (www.arpadi.de). Se incluyen más de 400 de las expresiones más frecuentes en las conversaciones entre médicos y pacientes y está disponible en 13 idiomas. 
Otros programas similares son Tooops66/77 y Patient55. Con estas herramientas, se pretende hacer más fluida la comunicación entre médicos y pacientes, así como facilitar el diagnóstico médico, sin que el idioma y la cultura supongan una barrera en situaciones en las que los idiomas distan mucho entre sí.

Sin embargo, no solo se buscan mejoras desde un punto de vista técnico, sino también desde una perspectiva social, ya que según me comunica la BDÜ el tema social está adquiriendo cada vez más importancia, y se está trabajando activamente en este asunto, ya que incluso se han organizado reuniones (especialmente en turco, debido al gran número de inmigrantes de este país en Alemania) para explicar a los extranjeros el funcionamiento de la sanidad en Alemania, y también se han elaborado publicaciones en las que se exponen los derechos de los pacientes (Bundesarbeitsgemeinschaft der Patientinnenstellen und Initiativen, BAGP) para perseguir este fin de informar al paciente.

\subsubsection{España}

El principal colaborador e informante directo ha sido el Hospital Ramón y Cajal, puesto que trabaja activamente en este tema, especialmente la Unidad de Medicina Tropical. Este hospital dispone de 18 personas en plantilla que disponen de formación específica para realizar traducciones e interpretaciones de los siguientes idiomas: inglés, francés, rumano, búlgaro, árabe, beréber, lenguas africanas, ruso y armenio. Normalmente, ofrecen servicios presenciales (es decir, un intérprete físico acude a la consulta), pero también trabajan a menudo con teléfonos de manos libres, así como con otros programas:

- Universal Doctor speaker: software multimedia para ayudar al profesional a entenderse con pacientes que hablen alguno de estos nueve idiomas: inglés, francés, alemán, portugués, ruso, rumano, árabe marroquí, chino mandarín y urdu. Creado por el Dr. Jordi Serrano Pons.

- Un servicio de teletraducción que sobre todo está en Urgencias. La empresa es Dualia.

- Si se necesita una persona en la consulta y que no tenemos intérprete, solicitamos a la ONG Accem en Madrid un intérprete. Ellos tienen un proyecto de interpretación en ámbito sanitario y está financiado por una entidad pública.

Correo electrónico recibido 
Además, este hospital trata de fomentar el aprendizaje de traducción e interpretación en el campo médico, y por ello, acoge a traductores e intérpretes en prácticas procedentes de un programa de posgrado para mediadores interculturales en los servicios de salud que organiza la Universidad de Alcalá de Henares, y cuyos fundamentos son:

- Analizar la calidad de la comunicación en los servicios públicos.

-Estudiar y contribuir al diseño de sociedades multiculturales.

-Desarrollar e intercambiar materiales para la formación.

Por otro lado, el Hospital Universitario de La Princesa presta los servicios de traducción e interpretación solo en la unidad de urgencias. Estos servicios los solicita el enfermo o el médico, y tan solo tienen que llamar por teléfono a una empresa privada (Dualia Teletraducciones, S.L.) que ofrece una interpretación telefónica. Según me informa este hospital por comunicación privada, dicha empresa presta sus servicios a varios hospitales de España (entre los que se incluyen todos los hospitales de la Comunidad de Madrid) así como a otros organismos públicos (por ejemplo, la policía). Se ofrece una amplia gama de los idiomas disponibles, y se fija un presupuesto anual para cubrir los costes derivados de este servicio.

Esta comunicación telefónica que es muy frecuente en los hospitales de la Comunidad de Madrid consiste en lo siguiente, según explica Dualia en su página web:

El sistema funciona a través de un teléfono "manos libres" compuesto de doble auricular y doble micrófono conectado a un teléfono móvil con el que dos personas de diferente cultura e idioma pueden mantener una conversación traducida en tiempo real por un intérprete no presencial que traduce desde el otro lado del hilo telefónico.

Por medio de una tecnología sencillísima de utilizar, eliminamos la necesidad de contratar a un intérprete presencial, al tiempo que borra las barreras lingüísticas y culturales.

El Hospital Universitario La Paz ofrece estos servicios también mediante interpretación telefónica. El proceso para la utilización del dispositivo de manos libres es similar a lo explicado anteriormente: también lo solicita el enfermo o el médico, y tan solo es necesario rellenar un cuestionario. Se ofrece siempre que se necesite (ya sea en urgencias o en consultas), y simplemente hay que llamar a un número móvil desde este terminal telefónico para que el paciente hable con un operador en su idioma (existe una amplia variedad) y pueda seleccionar el idioma apropiado. Después, se contacta con un intérprete y se establece una interpretación directa entre médico y paciente. 
En el Hospital San Camilo también se ofrecen estos servicios de traducción e interpretación, pero suele prestarlos la persona que está a cargo de la sección de Atención al Paciente, que es bilingüe de inglés. No obstante, también disponen de paneles y otros materiales en varios idiomas que pueden utilizarse durante las consultas. Además, al igual que en los casos anteriores, si se trata de idiomas desconocidos para el personal, utilizan el teléfono de manos libres para contactar con los intérpretes.

El Hospital Clínico San Carlos ofrece información telefónica las 24 horas del día en todos los servicios (urgencias, consultas, etc.). Aquí la interpretación también se ofrece a través del teléfono con manos libres de Dualia. En este caso, este servicio está disponible en 50 idiomas hasta las 18:00 y, partir de entonces, el número de idiomas se reduce.

Por último, según me informa por correspondencia privada el servicio de atención al ciudadano de la Comunidad de Madrid, dicha comunidad junto con alguna ONG y empresas farmacéuticas han adoptado medidas sociales, y han redactado folletos y otros materiales necesarios para difundir información sobre la sanidad pública a los inmigrantes.

En resumen, puede observarse que en España hay pocos hospitales que ofrezcan servicios presenciales de traducción e interpretación, pero sí se puede afirmar que la gran mayoría disponen de sistemas diseñados para eliminar estas barreras, y que hay un claro dominio del teléfono de manos libres de Dualia. No obstante, algún hospital (como es el caso del Ramón y Cajal) ofrece estos servicios en sus propias instalaciones, e incluso fomenta las prácticas y la formación de futuros mediadores lingüísticos y culturales. Asimismo, el hecho de que existan estudios de posgrado orientados hacia este asunto y que la Consejería de salud esté trabajando activamente en este tema indica que se trata de un tema actual y que está adquiriendo cada vez más importancia en el panorama español.

\subsection{INTÉRPRETES}

A raíz de las respuestas de los cuestionarios contestados (Anexo 4), puede afirmarse lo siguiente:

En primer lugar, hay que señalar que en la mayor parte de los casos, el inglés, el español y el alemán no tienen un papel demasiado significativo en lo que se refiere a interpretación en hospitales, ya que los primeros puestos los ocupan las lenguas del Este y lenguas africanas (este hecho está directamente relacionado con las migraciones).

En segundo lugar, es necesario resaltar que aunque la demanda del servicio de interpretación prevalece sobre la del servicio de traducción, también 
es necesario con frecuencia traducir folletos y otro tipo de documentación para pacientes.

Por otro lado, en cuanto a la forma de trabajo de los intérpretes, cabe destacar que la mayoría dispone permanentemente de un teléfono móvil o de un localizador para que se pueda contactar con ellos y solicitarles sus servicios. En base a lo expuesto anteriormente, es posible que el intérprete trabaje en el departamento de traducción e interpretación del hospital y que tenga que acudir de forma presencial a una consulta o al servicio de urgencias, o puede ser que la centralita de intérpretes le contacte para realizar una interpretación telefónica.

Entre las principales dificultades mencionadas en el desempeño de su trabajo, la mayoría destaca los factores sociales: informar al paciente sobre su enfermedad o tener que adaptar la información en función de su conocimiento del campo. En cuanto a los problemas lingüísticos, afirman que en su mayoría pueden resolverlos fácilmente preguntando directamente al médico, pidiéndole que reformule la frase o consultando rápidamente un diccionario en caso de duda; pero que nunca ha llegado a una situación de incomunicación o falta de entendimiento. Sin embargo, en el caso de los intérpretes que disponen de menos experiencia, sí se señalan algunos temores respecto a temas terminológicos, de contenido, comprensión dialectal, etc.

En relación con lo anterior, todos ellos opinan que es absolutamente necesario y recomendable disponer de una formación especializada y continua, tanto en una vertiente lingüística (idiomas, técnicas, código ético, características) como en una vertiente médica (terminología y contexto); por ello, muchos de ellos reciben formación complementaria en el hospital, en la empresa o por cuenta propia. Asimismo, casi todos los encuestados destacan la necesidad de fomentar estos servicios lingüísticos mediante la creación un departamento específico en cada hospital.

En el caso del Reino Unido, según indican los informantes, para poder trabajar como intérprete en los hospitales es necesario disponer de una titulación o bien tener 10 años de experiencia en el campo de la traducción médica. Además, hay hospitales que ofrecen su propia formación continua (como puede ser un curso de terminología médica). Por otro lado, los intérpretes encuestados en este país destacan la idea de que para mejorar el trabajo que realizan y obtener una mayor eficacia, debería instruirse a los médicos sobre cómo actuar cuando se trabaja con intérpretes; pero a su vez, también debería formarse a los intérpretes acerca de cómo reaccionar ante determinadas circunstancias, por ejemplo, cuando tienen que dar malas noticias o cuando ven cómo los pacientes se van deteriorando.

Según explicaba uno de los informantes anónimos en el cuestionario (intérprete alemán con experiencia en el sector), el proceso de interpretación empieza cuando el médico le llama por teléfono para acordar una cita en 
aproximadamente un cuarto de hora. De este modo, el intérprete puede prepararse para la conferencia y disponer del material que crea necesario. Este mismo intérprete afirma en su comunicado privado que una de las dificultades principales de este trabajo es que nunca sabe cómo se va a desarrollar la conversación y, por tanto, se desconoce el grado de especialización que se puede alcanzar. Por este motivo, no solo considera necesario disponer de conocimientos médicos, sino también tener información actualizada sobre la estructura organizativa del hospital. Por todo ello, apunta que para trabajar en este ámbito es muy útil disponer, por un lado, de una formación en técnicas interpretativas, y por otro, de una especialización médica, ya que facilita la interpretación en gran medida (al menos en lo que a comprensión se refiere). Asimismo, remarca las ventajas que supondría, según su opinión, contratar en algunos hospitales a personal fijo que realice estas tareas y que disponga de conocimientos médicos y lingüísticos, así como de conocimientos sobre técnicas traslativas e interpretativas.

En relación con esto, cabe destacar de nuevo la aportación que hace la BDÜ en el cuestionario contestado, ya que apunta que los servicios prestados en los hospitales no solo implican traducción, sino que a estos profesionales lingüísticos también se les encomiendan otras muchas tareas: resúmenes de textos especializados, elaboración de textos en lengua meta a partir de palabras clave, redacción de textos traducidos de forma automática, información de asuntos interculturales y en otros idiomas. Estos profesionales, ara llevar a cabo su trabajo, utilizan diversos materiales, entre los que destacan los siguientes: programas de tratamiento de textos, memorias de traducción, sistemas de tratamiento de terminología, programas de maquetación, etc.

Por último, puede extraerse también de varios cuestionarios, que en el caso de las consultas médicas, el intérprete debe ser totalmente fiel al contenido (aunque ello implique que quizás en ocasiones tenga que adaptar la terminología para que el paciente pueda comprenderlo). Es decir, lo importante en este caso no es la forma, sino el fondo, el mensaje en sí que le llega al paciente. Aunque el intérprete se vea obligado a reformular la frase para informar mejor al paciente, tiene que ser cauto e intentar ser lo más imparcial y precavido posible en lo que se refiere a aportar su opinión en la conversación.

\section{5. ¿ES NECESARIA LA ESPECIALIZACIÓN DEL TRADUCTOR / INTÉRPRETE MÉDICO?}

En relación con las respuestas de los informantes, puede decirse que hay una clara tendencia a favor de una especialización del traductor. Como he indicado previamente, la inmensa mayoría de pacientes, médicos e intérpretes 
opinan que el traductor debe especializarse, y consideran necesaria una formación tanto lingüística como médica.

Aunque esté más encaminada hacia el ámbito de la traducción, podría resultar útil la aportación y distinción que establece Josep Bonet (Bonet 2004: 37) sobre las escuelas de pensamiento: la de los traductores que proceden del mundo de la técnica (los "peritos con lenguas"), y la de la escuela de los que proceden del mundo de la traducción. Afirma asimismo que "la traducción técnica acostumbra a ser muy mala, porque exige una combinación de conocimientos que no suele darse en una sola persona”.

Por otro lado, destaca que todos los textos están cargados de marcas culturales, referentes históricos propios de una sociedad, señales reconocibles para los miembros de una cultura y posiblemente ajenos para los de otra cultura". Por ello, afirma que, "el traductor no deberá necesariamente traducir, modificar o adaptar todos estos elementos, pero forzosamente debería conocerlos" (Bonet 2004: 38).

A pesar de estas afirmaciones, Josep Bonet apunta también que la generación de un corpus documental permite hasta cierto punto que un traductor supla el desconocimiento que tiene sobre un tema y se dote de fraseología adecuada. Sin embargo, el problema es que "no siempre es posible crear corpus gigantescos y los corpus reducidos que se pueden construir fácilmente tienen una utilidad mucho más reducida" (Bonet 2004: 41).

\section{CONCLUSIONES}

En primer lugar, hay que mencionar que los tres países estudiados tienen una población multicultural importante que repercute claramente en las demandas de servicios de traducción e interpretación en los hospitales. En los tres casos estudiados, los servicios ofrecidos están estrechamente ligados a su legislación y el paciente tiene absoluto derecho a conocer su estado de salud, y por eso, este servicio de traducción e interpretación se considera un derecho básico del paciente.

A raíz de las aportaciones de los participantes, he podido comprobar que efectivamente, en mayor o menor medida, se prestan dichos servicios en casi todos los hospitales consultados, con un dominio general de la interpretación frente a la traducción. Asimismo, se ha podido observar que existen diversos tipos de interpretaciones en los tres países: interpretaciones telefónicas, interpretaciones de enlace presenciales e interpretaciones mediante programas informáticos.

Normalmente estos servicios están gestionados por la Consejería u otros organismos oficiales, que contratan a empresas privadas para que los 
presten en varios hospitales de la zona. La determinación del presupuesto para estos servicios puede variar entre hospitales: unos se basan en los datos de años previos, otros en las tasas de inmigración en la zona y otros abonan los cargos de los servicios cada vez que se presten.

Sin embargo, puede verse que se intenta frecuentemente ahorrar costes, tanto en el número de idiomas ofertados (manteniendo solo las lenguas más demandadas en cada hospital, o mediante la reducción de idiomas a partir de una determinada hora) como en personal, ya que a menudo esta labor la realizan otros profesionales del hospital con conocimientos de idiomas, o bien se efectúa por medio de máquinas u otros materiales que incluyen las principales frases utilizadas. Este último método de trabajo también está dando buenos resultados y cada vez se están desarrollando nuevas soluciones tecnológicas que permitan cubrir las necesidades (arpadi, Tooops66/77, Patient55, Dualia, Universal Doctor speaker, etc.). Asimismo, cada vez existen más grupos y organismos que investigan sobre este tema de actualidad para buscar soluciones y fomentar estos servicios en los hospitales (por ejemplo, el grupo FITISPos de Alcalá de Henares, la Universität Hamburg o el Hospital Ramón y Cajal, por citar algunos de ellos) y a la vez, también aumenta cada vez más la preocupación por explicar y difundir información sobre el sistema de salud a los inmigrantes.

Si el enfoque se establece, de forma más concreta, en el modo de trabajo preferido en cada país, puede afirmarse que en el Reino Unido se emplea frecuentemente tanto la interpretación presencial como la interpretación telefónica. Sin embargo, en España prima especialmente la interpretación telefónica (realizada la mayor parte de las veces por Dualia), aunque algunos hospitales, como el Ramón y Cajal, puedan tener también su propio departamento y disponer de personal en plantilla. En Alemania, por su parte, se ha podido observar que se suele recurrir normalmente a los profesionales sanitarios que hablan otros idiomas (interpretaciones presenciales), pero también trabajan con intérpretes contratados a través de la Krankenkasse (interpretaciones presenciales y telefónicas o con diferentes tipos de software).

Por último, en lo que se refiere a los cuestionarios respondidos, tanto médicos como pacientes opinan que estos servicios son de gran utilidad, ya que ayudan a que se desarrolle adecuadamente la comunicación en situaciones en las que, de lo contrario, sería imposible. Además, los pacientes lo consideran una gran ayuda y no piensan en ningún momento que pueda ser una violación de su intimidad. Asimismo, médicos, pacientes e intérpretes estiman necesaria una formación especializada del mediador lingüístico, ya que este no solo debe conocer lenguas, sino también la materia con la que trabaja. En relación con esta última idea, entre las principales dificultades que señalan los intérpretes, se 
encuentran los problemas terminológicos y de comprensión (de contenido o por causas de dialecto) en el caso de los principiantes o estudiantes en prácticas, y los problemas de registro, dificultades tecnológicas o incluso barreras emotivas, especialmente entre aquellos que cuentan con más experiencia o tratan repetidas veces con los mismos pacientes, ya que se pueden implicar demasiado en un caso y afectarles emotivamente.

A partir de todas estas conclusiones, se puede afirmar que en este campo aún queda un largo camino por recorrer y que existen muchos ámbitos relacionados en los que aún hay que investigar más a fondo porque muchos pacientes aún siguen teniendo grandes dificultades comunicativas. Podrían ser muchas las soluciones que se puedan implementar, no obstante, voy a indicar las que considero más importantes a partir de las conclusiones obtenidas:

En primer lugar, creo que es necesario invertir más en formación (tanto teórica como práctica); como acabo de mencionar más arriba, los intérpretes deben recibir una formación adecuada y específica en lo que se refiere a técnicas de interpretación (memorización, protocolo, estrategias, comportamiento, etc.), un nivel lingüístico alto en las dos lenguas que les permita comprender al médico y al paciente, así como una base de conocimientos médicos que les permita entender el contexto de la situación y poder cambiar el registro si fuera necesario. Esta formación es imprescindible porque el intérprete tiene que ser especialista en su trabajo, tanto en contenido (conocimientos médicos) como en forma (conocimientos expresivos y técnicas de traducción). Si se aplica esta idea sobre formación a las consultas médicas, se puede ver la importancia que tiene disponer de un conocimiento extralingüístico, es decir, de una formación médica que permita al intérprete comprender el mensaje sin problemas. Además, también se debe destacar en este apartado la imparcialidad que tiene que mostrar el intérprete, ya que él es sólo un mediador lingüístico y, por lo tanto, no debe ni dar su opinión ni intimidar al paciente.

En relación con esto, se debe apuntar que debido a la importancia que está adquiriendo la interpretación de enlace en el ámbito médico y la necesidad de profesionales que lleven a cabo esta labor, se ofrecen incluso programas de posgrado que permiten obtener esta especialización y proporcionan al futuro intérprete el conocimiento y las técnicas para desempeñar su trabajo con calidad.

En segundo lugar, también creo que es importante profundizar en la fase de investigación y elaboración de recursos y materiales, es decir, considero que cada vez será más necesario el hecho de que los hospitales dispongan de un listado de términos o expresiones en otros idiomas, de dibujos y elementos gráficos que puedan facilitar la explicación a pacientes extranjeros, etc. 
En tercer lugar, creo que sería conveniente que cada hospital dispusiera de su propio departamento de traducción e interpretación o un mínimo de traductores e intérpretes en plantilla, ya que de esta forma, se facilitaría la prestación de estos servicios y se evitaría en todo momento (incluso en situaciones de emergencia) que el paciente y el médico tuvieran problemas comunicativos. Dado que estos servicios son un campo bastante novedoso que está ligado a la creciente tasa de migraciones y frecuentes viajes e intercambios entre países, creo que a medida que aumente la demanda, será muy conveniente crear una sección específica que los gestione y los preste de forma eficaz.

En cuarto y último lugar, creo que sería conveniente fomentar el desarrollo de herramientas y programas informáticos que posibiliten la interpretación en aquellos hospitales o centros de salud en los que el número de pacientes extranjeros sea reducido, y no puedan por tanto disponer de un departamento específico. Actualmente, ya se está trabajando en ello y se están desarrollando nuevas herramientas como arpadi ${ }^{\circledR}$, Dualia o Universal Doctor speaker, por citar algunas de ellas.

\section{ANEXOS}

\subsection{ANEXO 1}

PATIENTS/PACIENTES/PATIENTEN

1. Name and surname/Nombre y apellidos/Vorname und Name:

2. Nationality/Nacionalidad/Staatsangehörigkeit:

3. Job/Cargo/Amt:

4. Email address/Dirección de correo electrónico/Email Adresse:

5. Since when do you live in Spain?/¿Desde cuándo vive en España?/Seit wann wohnen Sie in Spanien?: 
1. In which situation did you require the help of a translator or interpreter? / En qué caso necesitó la intervención de un traductor o intérprete? (Su experiencia) / Wann haben Sie die Hilfe von einem Übersetzer oder Dolmetscher gebraucht? (Ihre Erfahrung)

2. Was it useful? Why? / ¿Le resultó útil? ¿Por qué? / War es nötig? Warum?

3. Which are the main communication issues you would face on your own, if an interpreter or translator did not assist you? / ¿Cuáles son las principales dificultades comunicativas a las que se enfrentaría en el ámbito sanitario si no fuera por la intervención del traductor o intérprete? / Welche sind die kommunikativen Hauptschwierigkeiten für den Patienten, wenn kein Übersetzer oder Dolmetscher zur Verfügung steht?

4. Have you ever noticed the translator or interpreter had trouble understanding or making themselves understood? In case you have, what do you think are the main reasons? / ¿Ha observado falta de comprensión o expresión por parte del traductor o intérprete? En caso afirmativo, ¿a qué cree que se ha debido? / Haben Sie bemerkt, dass die Übersetzer oder Dolmetscher Verständnis- oder Ausdrucksschwierigkeiten hatten? Wenn ja, was waren, ihrer Meinung nach, die Gründe?

5. Do you think that translators and interpreters must receive medical training or medical translation training? / ¿Cree que es necesario que el traductor o intérprete disponga de una formación médica o de una formación en traducción especializada? / Finde Sie es notwendig, dass der Übersetzer oder Dolmetscher ein medizinisches Training, oder vielleicht ein Fachtraining in Übersetzung bekommt?

6. What would you recommend in order to achieve smoother communication and inform patients properly? / ¿Qué propuestas de mejora propone para que la comunicación sea más fluida y la información llegue mejor al paciente? / Was würden Sie empfehlen, um die Kommunikation flüssig zu gestalten, und dem Patienten die Information besser zu vermitteln? 
7. Do you think that the presence of a $3^{\text {rd }}$ party may violate your privacy? / ¿Vio invadida su intimidad? / Glauben Sie, dass die Anwesenheit Dritter eine Verletzung für die Privatsphäre darstellen kann?

8. Do you think there are any other inconveniences in these situations? / ¿Ha observado algún otro inconveniente? / Sind Ihnen noch weitere Probleme in diesen Situationen aufgefallen?

9. Other relevant data (personal experiences, useful information, etc.). / Cite otros datos relevantes (datos de interés, etc.). / Andere relevante Daten (persönliche Erfahrung, interessante Daten, etc.).

\title{
7.2. ANEXO 2
}

\section{PHYSICIANS/MÉDICOS/ÄRZTE}

\author{
Name and surname/Nombre y apellidos/Vorname und Name: \\ Job/Cargo/Amt: \\ Email address/Dirección de correo electrónico/Email Adresse:
}

1. How often do you see patients who do not speak English?/¿Con qué frecuencia atiende a pacientes que no hablen castellano?/Wie oft behandeln Sie Patienten, die nicht gut Deutsch sprechen?

2. Which nationality is the most frequent among the immigrants who need medical attention? Do you normally see German- and Spanishspeaking patients?/¿Qué nacionalidad es la más frecuente entre los inmigrantes que acuden al médico? ¿Atiende a muchos pacientes alemanes e ingleses?/Welche Staatsangehörigkeit haben die Einwanderer, die ins Krankenhaus gehen, am häufigsten? Behandeln Sie oft Spanier und Engländer?

3. How often do you need the services provided by cultural mediators (translators and interpreters)?/¿Con qué frecuencia recurre a intermediarios culturales (traductores o intérpretes)?/Wie oft brauchen Sie die Hilfe von Übersetzern oder Dolmetschern? 
4. In which cases do you specially need the presence of a cultural mediator (consultations, treatments, surgical procedures, emergency situations, ambulances, home visit, etc.)?/¿En qué casos se necesita especialmente la intervención de un mediador (consultas, tratamientos, operaciones, urgencia, ambulancias, visitas a domicilio, etc.)?/Wann brauchen Sie immer (oder sehr oft) die Hilfe von Übersetzern oder Dolmetschern (Praxis, Behandlung, chirurgischer Eingriff, Notaufnahme, Krankenwagen, Hausbesuch, etc.)?

5. Which are the main communication issues you face in these situations?/¿Cuáles son las principales dificultades comunicativas con las que se enfrenta en estos casos?/Welche sind die kommunikativen Hauptschwierigkeiten für den Arzt, wenn kein Übersetzer oder Dolmetscher zur Verfügung steht?

6. Have you found the help of a translator or interpreter useful?/¿Le ha resultado útil la intermediación de un traductor/intérprete?/Haben Sie die Hilfe von einem Übersetzer oder Dolmetscher für nötig gehalten?

7. Have you ever noticed the translator or interpreter had trouble understanding or making themselves understood? In case you have, what do you think are the main reasons?/¿Ha observado dificultades de comprensión por parte del traductor/intérprete? En caso afirmativo, ¿a qué cree que se han debido?/Haben Sie bemerkt, dass die Übersetzer oder Dolmetscher Verständnis- oder Ausdrucksprobleme hatten? Wenn ja, was waren, ihrer Meinung nach, die Gründe?

8. Do you think that translators and interpreters must receive medical training or medical translation training?/¿Cree que es necesario que el traductor/intérprete disponga de una formación médica o de una formación en traducción especializada?/Halten Sie es für nötig, dass der Übersetzer oder Dolmetscher ein medizinisches Training, oder vielleicht ein Fachtraining in Übersetzung bekommt?

9. What would you recommend in order to achieve smoother communication and inform patients properly?/¿Qué propuestas de mejora sugiere para que la comunicación sea más fluida y la información llegue mejor al paciente?/Was würden Sie empfehlen, um 
die Kommunikation flüssig zu gestalten, und dem Patienten die Information besser zu vermitteln?

10. Do you think that the presence of translators and interpreters may result in a violation of privacy?/ ¿Cree que la intervención de estos mediadores puede resultar en una violación de la privacidad?/Glauben Sie, dass die Anwesenheit Dritter eine Verletzung für die Privatsphäre darstellen kann?

11. Do you think there are any other inconveniences in these situations?/¿Observa algún otro inconveniente en estas situaciones?/Sind Ihnen noch weitere Probleme in diesen Situationen aufgefallen?

12. Other relevant data (personal experiences, useful information, etc.)./Otros datos relevantes (experiencias personales, datos de interés, etc.)./Andere relevante Daten (persönliche Erfahrung, interessante Daten, etc.).

\subsection{ANEXO 3}

CENTRO HOSPITALARIO / HOSPITAL CENTER / KRANKENHAUS

Name and surname/Nombre y apellidos/Vorname und Name:

Job/Cargo/Amt:

Email address/Dirección de correo electrónico/Email Adresse:

1. Does the hospital set an annual budget for translation/interpretation services, or does the cost have to be borne by patients?/¿El hospital destina un presupuesto fijo anual a los servicios de traducción e interpretación o se trata de un servicio que debe ser abonado por el propio paciente?/Legt das Krankenhaus ein jährliches Budget für Übersetzung und Dolmetschen fest, oder sollen die Patienten für diese Dienste selbst bezahlen? 
2. If the hospital sets a budget, which criteria are used to determine the amount?/En caso de que el hospital establezca un presupuesto, ¿en función de qué datos se fija esta cantidad?/Wenn das Krankenhaus ein jährliches Budget festlegen würde, worauf basiert diese Summe?

3. Has the hospital signed any agreement with any translation/interpretation agency, or does the hospital have its own inhouse translation/interpretation staff?/¿Han firmado algún contrato con alguna empresa de traducción/interpretación o disponen de una plantilla de traductores/intérpretes fija en el hospital?/Hat das Krankenhaus einen Vertrag mit einer Übersetzungs- oder Dolmetschensfirma geschlossen, oder hat das Krankenhaus sein einiges Stammpersonal in situ?

4. Which service (translation/interpretation) is more frequently requested? Please, explain which one receives higher funding and why./¿Qué servicio es más frecuente, traducción o interpretación? Explique en cuál se invierte más dinero y por qué./Welcher Dienst (Übersetzung oder Dolmetschen) wird am öftesten beantragt? Bitte, erklären Sie für welchen Dienst mehr Geld investiert wird und bitte, äußern Sie die Gründe dafür.

5. Is this service to be requested by a physician or by patients? $/$ Este servicio lo solicita el médico o el paciente?/Wird dieser Dienst durch den Arzt oder den Patienten beantragt?

\subsection{ANEXO 4}

TRANSLATORS and INTERPRETERS/TRADUCTORES e INTÉRPRETES/ÜBERSETZER und DOLMETSCHEN

Name and surname/Nombre y apellidos/Vorname und

Name:

Job/Cargo/Amt:

Email address/Dirección de correo electrónico/Email Adresse: 
1. How often do you have to perform any interpretation or translation in health sector?/¿Con qué frecuencia es necesario hacer alguna interpretación o traducción en el ámbito sanitario?/Wie oft müssen Sie als Übersetzer oder Dolmetschen im medizinischen Bereich arbeiten?

2. Which are the most demanded languages? Is it frequent to interpret or translate for German or Spanish patients?/¿Cuáles son los idiomas más demandados? ¿Es frecuente interpretar/traducir para en inglés y alemán?/Welche sind die Sprachen, die am häufigsten gefragt sind? Dolmetschen oder übersetzen Sie oft ins Spanische oder Englische im medizinischen Bereich?

3. Please, explain briefly how you organize your work (from the moment the hospital contacts you until you receive the money for the services provided)./Explique brevemente el modo en el que se organiza la prestación de este servicio, desde que el hospital se pone en contacto con usted hasta que se realiza el pago correspondiente./Bitte erklären Sie kurz wie die Arbeit organisiert wird (vom Zeitpunkt, an dem das Krankenhaus Sie kontaktiert, bis zum Moment in dem Sie das Geld für Ihre Dienste bekommen).

4. Have you ever had difficulties understanding or making yourself understood because the information was too technical? In case you have, which solutions did you find?/¿Ha tenido alguna vez dificultades de comprensión por términos técnicos? En caso afirmativo, ¿qué soluciones sigue en esos casos?/Haben Sie manchmal Verständnisoder Ausdrucksschwierigkeiten? Wenn ja, welche Lösung haben Sie ergriffen?

5. What are the main problems translators and interpreters face in a medical context?/¿Cuáles son los principales problemas a los que se enfrenta un traductor/intérprete en este campo?/Welche sind die Hauptprobleme, die sich einem Übersetzer oder Dolmetscher im medizinischen Bereich stellen?

6. What kind of documents are to be translated? Is the deadline usually acceptable?/¿Qué tipo de documentos se traducen? ¿Con qué plazo?/Welche Art von Dokumenten müssen übersetzen werden? Innerhalb von welcher Frist? 
7. Do you need specific training to be able to provide quality translation or interpretation?/¿Es necesario disponer de una especialización en el ámbito sanitario para traducir/interpretar con calidad?/Ist es nötig, ein Fachtraining im medizinischen Bereich zu haben, um qualitativ hochwertige Übersetzungen und Dolmetschen zu liefern?

8. What training should be compulsory for a medical translator?/¿Qué formación debería ser, en su opinión, obligatoria?/Welches Training sollte, Ihrer Meinung nach, verpflichtend sein?

9. Do you think it is recommended to have a specific training? $/ ¿$ Cree que sería recomendable disponer de una formación específica?/Ihrer Meinung nach, ist es nötig, ein medizinisches Training oder Fachtraining in Übersetzung zu bekommen?

10. What would you recommend in order to improve communication and to get proper information?/¿Qué mejoras propondría para un óptimo funcionamiento de este servicio?/Was würden Sie empfehlen, um eine optimale Dienstleistung zu erreichen?

11. Other relevant data (personal experiences, useful information, etc.)./Otros datos relevantes./Andere relevante Daten (persönliche Erfahrung, interessante Daten, etc.).

\section{BIBLIOGRAFÍA}

\subsection{FUENTES ELECTRÓNICAS}

Accem, www.accem.es. Consulta realizada el 05/04/14.

Amt für Statistik und Wahlen Landeshauptstadt Düsseldorf, http://www.duess eldorf.de/statistik/d_ueberblick/gesamt.shtml. Consulta realizada el 20/03/14.

Arpadi, www.arpadi.de. Consulta realizada el 01/06/09.

BAGP, http://www.gesundheits.de/bagp/BAGP-Dokumente/praepmigr.pdf. Consulta realizada el 15/07/14.

BBC, http://news.bbc.co.uk/1/shared/spl/hi/uk/05/born_abroad/html/ove rview.stm. Consulta realizada el 25/03/14.

Bundesministerium des Innern, https://www.bmi.bund.de/cln_144/DE/Hom e/startseite_node.html. Consulta realizada el 20/03/14. 
CiberCaixa, http://obrasocial.lacaixa.es/cibercaixa/programa es.html. Consult a realizada el 05/06/14.

COMRADE, www.comrade.es. Consulta realizada el 05/04/14.

Comunidad de Madrid, http://www.publicaciones-isp.org/index.asp. Consulta realizada el 10/05/14.

Consulado de España en el Reino Unido, http://www.conspalon.org/. Consulta realizada el 26/03/14.

Cruz roja, http://www.cruzroja.es/preportada/tv/index.html. Consulta realizada el 05/04/14.

Departamento de Comunicación de la Fundación BBVA, www.fbbva.es/TLF U/dat/np_demograf_extranjeros.doc. Consulta realizada el 25/03/14.

Detastis, http://www.destatis.de/jetspeed/portal/cms/Sites/destatis/Internet /DE/Content/Statistiken/Bevoelkerung/AuslaendischeBevoelkerung/Tab ellen/Content50/TOP10, templateId=renderPrint.psml. Consulta realizada el $07 / 03 / 14$

Deutsche-mittelgebirge, http://www.deutsche-mittelgebirge.de/Info_Bevoelke rungsdichte.htm. Consulta realizada el 20/03/14.

DUALIA, http://www.dualia.es/index.php?main=empresa. Consulta realizada el 05/08/14.

El Mundo, http://estaticos.elmundo.es/elmundo/2003/graficos/jul/s1/inmig racion.gif. Consulta realizada el 20/03/14.

Embajada de Alemania, http://www.madrid.diplo.de/Vertretung/madrid/es/S tartseite.html. Consulta realizada el 25/03/14.

Embajada de Reino Unido, http://ukinspain.fco.gov.uk/es. Consulta realizada el 26/03/14.

Foreign and Commonwealth Office (FCO), http://www.fco.gov.uk/en/. Consulta realizada el 30/03/14.

Formación e investigación en Traducción e Interpretación en los Servicios Públicos, http://www.fitispos.com.es/index2.html. Consulta realizada el 27/07/14.

Fundación de Ciencias de la Salud, http://geopress.educa.aragon.es/WebgeoN EW/libro/poblacion/Mapas/Mapa_inmigrantesCCAA.jpg. Consulta realizada el 15/04/14.

Fundación de Ciencias de la Salud, http://www.fcs.es. Consulta realizada el $15 / 04 / 14$.

Geopress, http://geopress.educa.aragon.es/WebgeoNEW/libro/poblacion/5i nmigracion.pdf. Consulta realizada el 20/04/14.

German Embassy London, http://www.london.diplo.de/Vertretun/london/e n/Startseite.html. Consulta realizada el 27/03/14.

Home Office, http:/ / www.official-documents.gov.uk/document/cm69/6904/ 6904.pdf. Consulta realizada el 05/03/14. 
Home Office (Research Development Statistics), http:/ /www.homeoffice.gov. uk/passports-and-immigration/immigration/. Consulta realizada el $05 / 03 / 14$.

Home Office (UK Border Agency), http://www.ukba.homeoffice.gov.uk/. Consulta realizada el 05/03/14.

Information und Technik Nordrhein-Westfalen, http://www.it.nrw.de/cgi$\mathrm{bin} /$ search.cgi? $\mathrm{q}=$ Einb $\%$ FCrgerungen\&form $=$ extended $\& \mathrm{~m}=$ all $\& w \mathrm{~m}={ }_{\mathrm{wrd}}$. Consulta realizada el 07/03/14.

Information und Technik Nordrhein-Westfalen, http://www.it.nrw.de/presse/ pressemitteilungen/2008/pdf/einbuergerungen_2000_2007.pdf. Consulta realizada el $07 / 03 / 14$.

Information und Technik Nordrhein-Westfallen, http:/ /www.it.nrw.de/statisti $\mathrm{k} / \mathrm{a} /$ daten/amtlichebevoelkerungszahlen/rp1_juni08.html.

Consulta realizada el 07/03/14.

Institute for Public Policy Research, http://www.ippr.org.uk/publicationsandr eports $/$ publications.asp?title $=\&$ author $=\&$ pubdate $=\&$ theme $=$ Migration + an $\mathrm{d}+$ Integration\&search $=$ search. Consulta realizada el 10/03/14.

Instituto Nacional de Estadística (INE), http:/ /www.ine.es/jaxi/tabla.do?path $=/ \mathrm{t} 20 / \mathrm{e} 245 / \mathrm{p} 05 / \mathrm{a} 2008 / 10 / \&$ file $=00028003$.px\&type $=$ pcaxis $\& \mathrm{~L}=0$. Consulta realizada el 15/03/14.

Instituto Nacional de Estadística (INE), http://www.ine.es/inebmenu/mnu_ci fraspob.htm. Consulta realizada el 15/03/14.

Instituto Nacional de Estadística (INE), http:/ /www.ine.es/jaxi/tabla.do?path $=/ \mathrm{t} 20 / \mathrm{e} 245 / \mathrm{p} 04 / \mathrm{provi} / 10 / \&$ file $=0$ ccaa007.px\&type $=$ pcaxis $\& \mathrm{~L}=0$. Consulta realizada el 15/03/14.

Instituto Nacional de Estadística (INE), http:/ /www.ine.es/jaxi/tabla.do?path $=/ \mathrm{t} 20 / \mathrm{p} 319 / \mathrm{a} 2007 / \mathrm{p} 02 / 10 / \&$ file $=04001 \cdot \mathrm{px} \&$ type $=$ pcaxis\&L $=0$. Consulta realizada el 15/03/14.

Instituto Nacional de Estadística (INE), http:/ /www.ine.es/jaxi/tabla.do?path $=/ \mathrm{t} 20 / \mathrm{e} 260 / \mathrm{a} 2008 / 10 / \&$ file $=$ ca001.px\&type $=$ pcaxis $\& \mathrm{~L}=0 . \quad$ Consulta realizada el 15/03/14.

Instituto Nacional de Estadística (INE), http:/ /www.ine.es/jaxi/tabla.do?path $=/ \mathrm{t} 20 / \mathrm{e} 260 / \mathrm{a} 2008 / 10 / \&$ file $=$ mun28.px\&type $=$ pcaxis $\& \mathrm{~L}=0 . \quad$ Consulta realizada el 15/03/14.

Instituto Nacional de Estadística (INE), http:/ /www.ine.es/jaxi/tabla.do?path $=/ \mathrm{t} 20 / \mathrm{e} 245 / \mathrm{p} 04 / \mathrm{provi} / 10 / \&$ file $=0 \mathrm{ccaa003}$. PX\&type $=$ pcaxis $\& \mathrm{~L}=0$.

Consulta realizada el 15/03/14.

Instituto Nacional de Estadística (INE), http://www.ine.es/prensa/np503.pdf. Consulta realizada el 15/03/14.

Ministerio de Asuntos Exteriores y Cooperación, http://www.maec.es/es/Ho me/Paginas/HomeEs.aspx. Consulta realizada el 23/03/14. 
Ministerio de Educación y Ciencia, http://sauce.pntic.mec.es/jotero/Inmigra/ Cuantos.htm. Consulta realizada el 23/03/14.

Ministerio de Sanidad y Consumo, http://www.msc.es/organizacion/sns/libro SNS.htm. Consulta realizada el 29/05/14.

Ministerio del Interior, http://www.mir.es/MIR/Directorio/Servicios_Periferi cos/Cuerpo_Nacional_de_Policia/Oficinas_de_extranjeros/Madrid/.

Consulta realizada el 23/03/14.

Munimadrid, http://www.munimadrid.es/portal/site/munimadrid/menuitem. $199479 \mathrm{e} 61 \mathrm{~b} 01 \mathrm{~b} 0 \mathrm{aa} 7 \mathrm{~d} 245 \mathrm{f019fc08a0c} /$ ?vgnextoid=61 ae140630b5f110 VgnV CM2000000c205a0aRCRD\&vgnextchannel=6c51adb6fc3d8010VgnVCM10 0000dc0ca8c0RCRD. Consulta realizada el 29/05/14.

Munimadrid, http://www.munimadrid.es/portal/site/munimadrid/menuitem. 8b2184148b70b0aa7d245f019fc08a0c/?vgnextoid =6fe9e56e77010210 VgnV CM2000000c205a0aRCRD\&vgnextchannel=d33d9ad016e07010VgnVCM1 00000dc0ca8c0RCRD. Consulta realizada el 29/05/14.

Munimadrid, http://www.munimadrid.es/portal/site/munimadrid/menuitem. 8b2184148b70b0aa7d245f019fc08a0c/?vgnextoid=03aa61e899c63110VgnV CM1000000b205a0aRCRD\&vgnextchannel=d33d9ad016e07010VgnVCM1 00000dc0ca8c0RCRD. Consulta realizada el 29/05/14.

Munimadrid, http://www.munimadrid.es/UnidadesDescentralizadas/UDCEst adistica/Publicaciones / PoblacionExtranjera/1Enero2009/Boletín\%20Extra njeros\%2009f.pdf. Consulta realizada el 29/05/14.

National Statistics, http://www.statistics.gov.uk/cci/nscl.asp?ID=7512. Consulta realizada el 20/03/14.

National Statistics, http://www.statistics.gov.uk/pdfdir/ppmg1108.pdf. Consulta realizada el 20/03/14.

National Statistics, http:/ / www.statistics.gov.uk/statbase/Product.asp?vlnk=1 5147. Consulta realizada el 20/03/14.

National Statistics, http://www.statistics.gov.uk/statbase/Product.asp?vlnk=1 5106.Consulta realizada el 20/03/14.

NHS, http://www.nhs-translation.com/index.htm. Consulta realizada el $30 / 05 / 14$.

NHS, http://www.nhs-translation.com/services.htm. Consulta realizada el $30 / 05 / 14$.

NHS London, http://www.london.nhs.uk/your-nhs-in-london/structureexplained. Consulta realizada el 20/03/14.

Obra social Fundación La Caixa, http://obrasocial.lacaixa.es/apl/actividades/a ctivitats.actividad_es.html?idActividad $=11640 \& J S E S S I O N I D=C q 5 R d K e Z$ 9oxlYIPX9_1LWiu. Consulta realizada el 27/04/14. 
Obra social Fundación La Caixa, http:/ / obrasocial.lacaixa.es/apl/actividades/a ctivitats.actividad_es.html?idActividad $=20510 \& J S E S S I O N I D=C q 5 \mathrm{RdKeZ}$ 9oxlYIPX9_1LWiu. Consulta realizada el 27/04/14.

Office for National Statistics (ONS), http://www.statistics.gov.uk/cci/nugget. asp?ID $=6$. Consulta realizada el 20/03/14.

Office for National Statistics (ONS), http://www.statistics.gov.uk/statbase/ss dataset.asp?vlnk=9664\&More $=$ Y. Consulta realizada el 20/03/14.

Office for National Statistics (ONS), http://www.statistics.gov.uk/statbase/ss dataset.asp? $\operatorname{vlnk}=8271 \&$ More $=$ Y. Consulta realizada el 20/03/14.

Office for National Statistics (ONS), www.statistics.gov.uk/pdfdir/ppmg0509. pdf. Consulta realizada el 20/03/14.

Red Acoge, http://www.redacoge.org/. Consulta realizada el 05/04/14.

Revista

Médicos, http://www.revistamedicos.com.ar/numero10/pagina07.htm. Consulta realizada el 29/05/14.

Schaumburger Nachrichten, http://www.sn-online.de/Nachrichten/Hannover /Uebersicht/Krankenkasse-auf-Tuerkisch. Consulta realizada el 15/07/14.

Secretaría de Estado de Inmigración y Emigración, http:/ / extranjeros.mtin.es/ es/index.html. Consulta realizada el 23/03/14.

South East England Regional Assembly, http://www.southeast-ra.gov.uk/doc uments/monitoring_success/sed16-mar09.pdf. Consulta realizada el 23/03/14.

Spanisches Konsulat in Düsseldorf, http://www.konsulate.de/info/info_spani sches_konsulat_duesseldorf_deutschland.php. Consulta realizada el 23/03/14.

Statistisches Amt, Direktorium, Landeshauptstadt München, http://www.msta tistik-muenchen.de/themen/bevoelkerung/jahreszahlen/jahreszahlen_2007 /p_jt080111.pdf. Consulta realizada el 23/03/14.

Statistisches Bundesamt Deutschland, http://www.destatis.de/jetspeed/portal /cms/Sites/destatis/Internet/DE/Content/Statistiken/Bevoelkerung/Ausl aendischeBevoelkerung/Tabellen/Content75/EinbuergerungStaatsangehoe rigkeit,templateId=renderPrint.psml. Consulta realizada el 28/03/14.

Statistisches Bundesamt Deutschland, http://www.statistik-portal.de/StatistikPortal/de_jb01_jahrtab2.asp. Consulta realizada el 28/03/14.

Statistisches Bundesamt Deutschland, http://www.destatis.de/jetspeed/portal /cms/Sites/destatis/Internet/DE/Navigation/Statistiken/Bevoelkerung/A uslaendischeBevoelkerung/Tabellen.psml. Consulta realizada el 28/03/14.

Statistisches Bundesamt Deutschland, http://www.destatis.de/jetspeed/portal /cms/Sites/destatis/Internet/DE/Navigation/Statistiken/Bevoelkerung/B evoelkerungsstand/Bevoelkerungsstand.psml. Consulta realizada el $23 / 03 / 14$. 
Statistisches Bundesamt Deutschland, http://www.destatis.de/jetspeed/portal /cms/Sites/destatis/Internet/DE/Content/Statistiken/Zeitreihen/LangeR eihen/Bevoelkerung/Content100/lrbev02a,templateId=renderPrint.psml. Consulta realizada el 23/03/14.

Thüringer Landesamt für Statistik, http:/ / www.thueringen.de/imperia/md/co ntent/tsk/ab/ausl_nder_in_th_ringen_2008.pdf. (un mapa muy interesante). Consulta realizada el 25/03/14.

Tooops, http://www.pflege-fremdsprachen-system-rossow.eu/. Consulta realizada el 15/07/14.

UK in Germany, http://ukingermany.fco.gov.uk/de. Consulta realizada el $27 / 03 / 14$.

UK National Statistics, http://www.statistics.gov.uk/about/data/methodology /specific/population/future/imps/updates/downloads/STM_mid07.pdf. Consulta realizada el 27/03/14.

UK National Statistics, http:/ / www.statistics.gov.uk/census2001/pop2001/bri ghton_and_hove_ua.asp. Consulta realizada el 27/03/14.

UK National Statistics, http:/ /www.statistics.gov.uk/hub/population/migratio $\mathrm{n} /$ migration-within-the-uk/index.html. Consulta realizada el 270/03/14.

UK National Statistics, http:/ / www.statistics.gov.uk/hub/population/migratio n/international-migration/index.html. Consulta realizada el 27/03/14.

UK University of Kentucky, http:/ / portal.hosp.uky.edu/evpha/ops/ha/qualit y/interpreters/default.aspx.Consulta realizada el 27/03/14.

UNESCO, http://whc.unesco.org/en/statesparties/gb. Consulta realizada el 06/05/14.

Universal Doctor, http://www.universaldoctor.com/. Consulta realizada el 30/05/14.

UNIVERSITAT ROVIRA I VIRGILI, http:/ /isg.urv.es/library/papers/grau.htm. Consulta realizada el 27/03/14.

\subsection{FUENTES IMPRESAS}

Bonet, J. (2004). «La necesaria especialización del traductor técnico» en Gonzalo García, C. y García Yebra, V. (eds.): Documentación y terminología para la traducción especializada. Madrid: Arco/Libros, pp. 37-48.

Collados Aís, Á. y Fernández Sánchez, M. (coord.) (2001). Manual de interpretación bilateral. Granada: Comares.

Gamero, S. (2001). La traducción de textos técnicos. Barcelona: Ariel.

Melander, B (1998). «Culture or genre? Issues in the interpretation of crosscultural differences in scientific papers» en Fortanet, I., S. Posteuillo, J.C. 
La necesidad de servicios de Traducción e Interpretación en el sector sanitario

Palmer y J.F. Coll (eds). Genre Studies in English for Academic Purposes. Castelló: Publicacions Uiversitat Jaume I, pp. 211-226.

Navarro, F. (1997). Traducción y lenguaje en medicina. Barcelona: Fundación Dr. Antonio Esteve.

Valero Garcés, C. (ed.) (2003). Traducción e interpretación en los servicios públicos: contextualización, actualidad y futuro. Granada: Comares. 